\title{
Histopathological comparison of Kearns-Sayre syndrome and PGC-1 $\alpha$-deficient mice suggests a novel concept for vacuole formation in mitochondrial encephalopathy
}

\author{
Levente Szalardy ${ }^{1}$, Mate Molnar ${ }^{1}$, Rita Torok ${ }^{1}$, Denes Zadori ${ }^{1}$, Laszlo Vecsei ${ }^{1,2}$, Peter Klivenyi ${ }^{1}$, Pawet Piotr Liberski ${ }^{3}$, \\ Gabor Geza Kovacs ${ }^{4}$ \\ ${ }^{1}$ Department of Neurology, Faculty of Medicine, Albert Szent-Györgyi Clinical Center, University of Szeged, Szeged, Hungary, \\ ${ }^{2}$ MTA-SZTE Neuroscience Research Group, Szeged, Hungary, ${ }^{3}$ Department of Molecular Pathology and Neuropathology, Chair of Oncology, \\ Medical University of Lodz, Lodz, Poland, ${ }^{4}$ Institute of Neurology, Medical University of Vienna, Vienna, Austria
}

\begin{abstract}
Despite the current hypotheses about myelinic and astrocytic ion-dyshomeostasis underlying white (WM) and grey matter (GM) vacuolation in mitochondrial encephalopathies, there is a paucity of data on the exact mechanism of vacuole formation. To revisit the concepts of vacuole formation associated with mitochondrial dysfunction, we performed a comparative neuropathological analysis in Kearns-Sayre syndrome (KSS) and full-length peroxisome proliferator-activated receptor- $\gamma$ coactivator-1 $\alpha$ (FL-PGC-1 $\alpha$ )-deficient mice, a recently proposed morphological model of mitochondrial encephalopathies. Brain tissues from an individual with genetically proven KSS (22-year-old man) and aged FL-PGC-1 $\alpha$-deficient and wild-type (male, 70-75-week-old) mice were analysed using ultrastructural and immunohistochemical methods, with a specific focus on myelin-related, oligodendroglial, axonal and astrocytic pathologies. Besides demonstrating remarkable similarities in the lesion profile of KSS and FL-PGC-1 $\alpha$-deficient mice, this study first provides morphological evidence for the identical origin of WM and GM vacuolation as well as for the presence of intracytoplasmic oligodendroglial vacuoles in mitochondriopathies. Based on these observations, the paper proposes a theoretical model for the development of focal myelin vacuolation as opposed to the original concepts of intramyelin oedema. Placing oligodendrocytes in the centre of tissue lesioning in conditions related to defects in mitochondria, our observations support the rationale for cytoprotective targeting of oligodendrocytes in mitochondrial encephalopathies, and may also have implications in brain aging and multiple sclerosis, as discussed.
\end{abstract}

Key words: Kearns-Sayre syndrome, PGC-1 $\alpha$, mitochondrial encephalopathy, vacuole, myelin, oligodendrocyte.

\section{Introduction}

Mitochondrial diseases comprise a group of inherited or sporadic neurological disorders in which proper mitochondrial function is compromised. Either the mitochondrial or nuclear genome is involved by the genetic alterations. Based on the symptomatic appearance, such diseases are divided into characteristic syndromes, including MELAS (mitochondrial

\section{Communicating author}

Prof. Gabor Geza Kovacs, Institute of Neurology, Medical University of Vienna, Währinger Gürtel 18-20, 1090 Vienna, Austria,

e-mail: gabor.kovacs@meduniwien.ac.at 
encephalomyopathy, lactic acidosis, and stroke-like episodes), MERRF (myoclonic epilepsy with ragged red fibres), LHON (Leber's hereditary optic neuropathy), MNGIE (mitochondrial neurogastrointestinal encephalopathy), NARP (neuropathy, ataxia, retinitis pigmentosa), Leigh's syndrome and Kearns-Sayre syndrome (KSS) $[4,6,9,47]$, as well as the more recently characterized LBSL (leukoencephalopathy with brainstem and spinal cord involvement and lactate elevation) $[39,48]$ and HBSL (hypomyelination with brainstem and spinal cord involvement and leg spasticity) [44]. The leading symptoms develop due to the progressive pathology in organs with high energy demand, resulting usually in early death. Though the distribution of neuropathological alterations is characteristic of a particular syndrome, all mitochondrial encephalopathies present in various degrees of vacuolation in the white (WM) and grey matter (GM), regional neurodegeneration with reactive astrogliosis and, less generally, capillary proliferation $[4,6,9,47]$.

Recently, mice deficient in the expression of fulllength peroxisome proliferator-activated receptor gamma coactivator-1 $\alpha$ (PGC-1 $\alpha$ ) protein (FL-PGC-1 $\alpha$ ) have been suggested as a morphological model for mitochondrial diseases [43]. PGC-1 $\alpha$ is a nuclear-encoded protein that plays important roles in the transcriptional regulation of mitochondrial function at several levels, including mitochondrial biogenesis, glucose and lipid metabolism as well as oxidative stress defence, and its dysfunction has been implicated in the pathogenesis of a number of neurodegenerative diseases both in humans and experimental animals $[37,43]$. FL-PGC- $1 \alpha$-deficient mice develop liver disease, decreased locomotion and muscle weakness [23], together with a spongiform leukoencephalopathy with wide-spread vacuolation accompanied by reactive astrogliosis in the brainstem and the cerebellar nuclei, resembling the neuropathological alterations seen in KSS [43].

According to the current notion, WM vacuoles in mitochondrial encephalopathies are linked to intramyelin 'oedema' due to preferential mitochondrial dysfunction in oligodendrocytes, presenting as intramyelin 'bubbles' due to splitting of the myelin sheath at the intraperiod line. In contrast, GM neuropil vacuoles are presumed to develop because of the failure of ATP-dependent ion transporters in astrocytic membranes $[32,40,46]$. While intraperiod line splitting is a common finding in cases with status spongiosus, the mechanism through which focal and circumscribed fluid accumulation develops in an extracellular compartment is only poorly understood. Thus, our knowledge about the origin of intramyelin and neuropil vacuolation remains speculative.

Our first study on FL-PGC-1 $\alpha$-deficient mice indicated that the majority of the observed vacuoles were associated with myelin in the absence of apparent axonal involvement [43]. Based on these observations, we aimed to evaluate the origin and nature of vacuole formation in aged FL-PGC- $1 \alpha$-deficient mice, in comparison with a human case of KSS. Our results emphasize the novel pathogenic role of the oligodendrocytes in the formation of both WM and GM vacuoles. Besides providing a better understanding of tissue lesioning in mitochondrial encephalopathies, our observations may also have implications for WM damage in multiple sclerosis as well as in the aging brain, related pathologies where mitochondrial dysfunction may also play key roles in the pathogenesis.

\section{Material and methods \\ Patient and animals}

Brain tissue of a male KSS patient with a $4.9 \mathrm{kbp}$ common deletion in mtDNA died at 22 years of age was used for neuropathological analysis. The mother gave informed consent prior to the neuropathological work-up. The study was adhered to the tenets of the most recent revision of the Declaration of Helsinki.

For comparison, 70-75-week-old FL-PGC-1 $\alpha$-deficient and age-matched wild-type C57Bl/6J male mice were involved in this study. The animals were housed in cages (maximum 4 per cage) in standard conditions with 12-12 h light-dark cycle and ad libitum access to standard pellet food and water. The experiments were performed in accordance with the European Communities Council Directive (86/609/EEC) and were approved by the local Animal Care Committee.

\section{Immunohistochemistry and histology}

Formalin-fixed paraffin-embedded blocks of different regions of the KSS brain, including several neocortical areas, basal ganglia, thalamus, hippocampus, amygdala, brainstem and cerebellum, as well as a double hemispheric block at the levels of WM lesions were examined. The murine brains were removed on ice and halved at the midline immediately following decapitation. Half brains were fixed 
in $4 \%$ paraformaldehyde overnight and kept in $15 \%$ glycerol in $4^{\circ} \mathrm{C}$ until embedding in paraffin. The other halves of the murine brains were used for the frozen sections (i.e. for Oil Red O staining).

3- $\mu \mathrm{m}$-thick sections were stained with KlüverBarrera (Luxol and Fast red) and Oil Red O stainings. For immunohistochemistry we applied the following monoclonal antibodies (cross-reacting with mouse): anti-amyloid precursor protein (APP) (1: 500, Millipore, Billerica, Mass., USA), phosphorylated and non-phosphorylated neurofilaments (clones SMI-31 and SMI-32, markers of axons and neuronal cell bodies; 1 : 5000 and 1 : 200, respectively, Covance, Berkeley, CA, USA), TPPP/p25 (1 : 2000; a marker of mature oligodendrocytes [15]) and microtubule associated protein-2 (MAP-2, marker of neuronal cell bodies and dendrites; Millipore). Furthermore, the following polyclonal antibodies were used: antiglial fibrillary acidic protein (GFAP; 1 : 3000, Dako, Glostrup, Denmark), MBP (myelin basic protein; 1 : 400, Dako) and Iba1 (1 : 1000, Wako Chemicals, Osaka, Japan). The DAKO EnVision detection kit, peroxidase/DAB, rabbit/mouse (Dako) was used for visualization of antibody reactions. When applying mouse antibodies, we used the M.O.M. kit (Vector Laboratories, Burlingame, Calif., USA) to prevent the aspecific background staining of endogenous mouse immunoglobulins.

\section{Electron microscopy}

Human KSS samples from the basal ganglia (internal capsule) and WM lesions were immersion-fixed in $4 \%$ glutaraldehyde for 3 days. The animals used for electron microscopy were anaesthetised with isoflurane and were perfused transcardially with modified Hamori fixative $(1.5 \%$ glutaraldehyde and $1 \%$ formaldehyde in phosphate buffer) for 18 min with a $10 \mathrm{ml} /$ min flow, prior to decapitation and subsequent sample preparation.

The obtained central nervous system (CNS) samples were postfixed in $1 \%$ osmium tetroxide for 1-2 $\mathrm{h}$, dehydrated through a series of graded ethanols and propylene oxide, and then embedded in Embed 812 resin (Electron Microscopy Sciences, EMS 14120). Semi-thin sections were stained with toluidine blue, blocks trimmed, and ultrathin sections stained with lead citrate and uranyl acetate. Specimens were examined using a JEM-100C transmission electron microscope.

\section{Results \\ Comparison of lesion profiles}

\section{Vacuolation}

Klüver-Barrera staining revealed a widespread spongiform change in both the KSS brain and FL-PGC-1 $\alpha$-deficient mice. In both, the vacuolation predominated in the WM; however, vacuoles in the GM neuropil were also observed with apparently lower frequency (Fig. 1A-F). The vacuolation commonly affected the internal capsule, striatal pencil fibres, cerebellar WM, thalamic fascicules, pyramidal tracts and, less intensively, the corpus callosum. Vacuoles were commonly present in the neuropil, most intensively in the brainstem, but also consistently present in the basal ganglia, thalamic nuclei and less intensively in the neocortex, where they showed a predilection toward the deep cortical layers. The severity of vacuolation was more prominent in the KSS brain than in experimental animals; in some regions with coarse cystic-necrotic lesions and myelin pallor, while in some others with demyelinated foci (e.g. postcentral region, cerebellar WM, some of the pencil fibres) (Fig. $1 \mathrm{C}$ and F). Demyelination and cystic-necrotic lesions were undetected in FL-PGC-1 $\alpha$-deficient mice. Notably, the examined aged wild-type brains presented vacuoles showing similar appearance and distribution as their FL-PGC-1 $\alpha$-deficient counterparts; however, their frequency was remarkably lower in all examined regions (Fig. 1A-B, D-E).

\section{Astrogliosis}

GFAP staining revealed moderate to severe reactive astrogliosis in the brainstem and cerebellum of both the KSS and the FL-PGC-1 $\alpha$-deficient brains (Fig. 1G-I). In the KSS brain, astroglial reaction was observed in the tectal midbrain, the area of the inferior olive in the medulla oblongata, the dentate nucleus of the cerebellum and the Purkinje cell layer (Bergmann gliosis). In the FL-PGC-1 $\alpha$-deficient mice, severe reaction was present in the medulla oblongata and the pons often in a confluent pattern involving large areas without being limited to certain groups of nuclei, whereas mild reaction with patchy astrocytosis was observed in the midbrain and in the deep cerebellar nuclei. Notably, the caudate-putamen of PGC-1 $\alpha$-deficient mice were free of astroglial reaction even at this age (Fig. 2), supporting our prior observations [43]. 

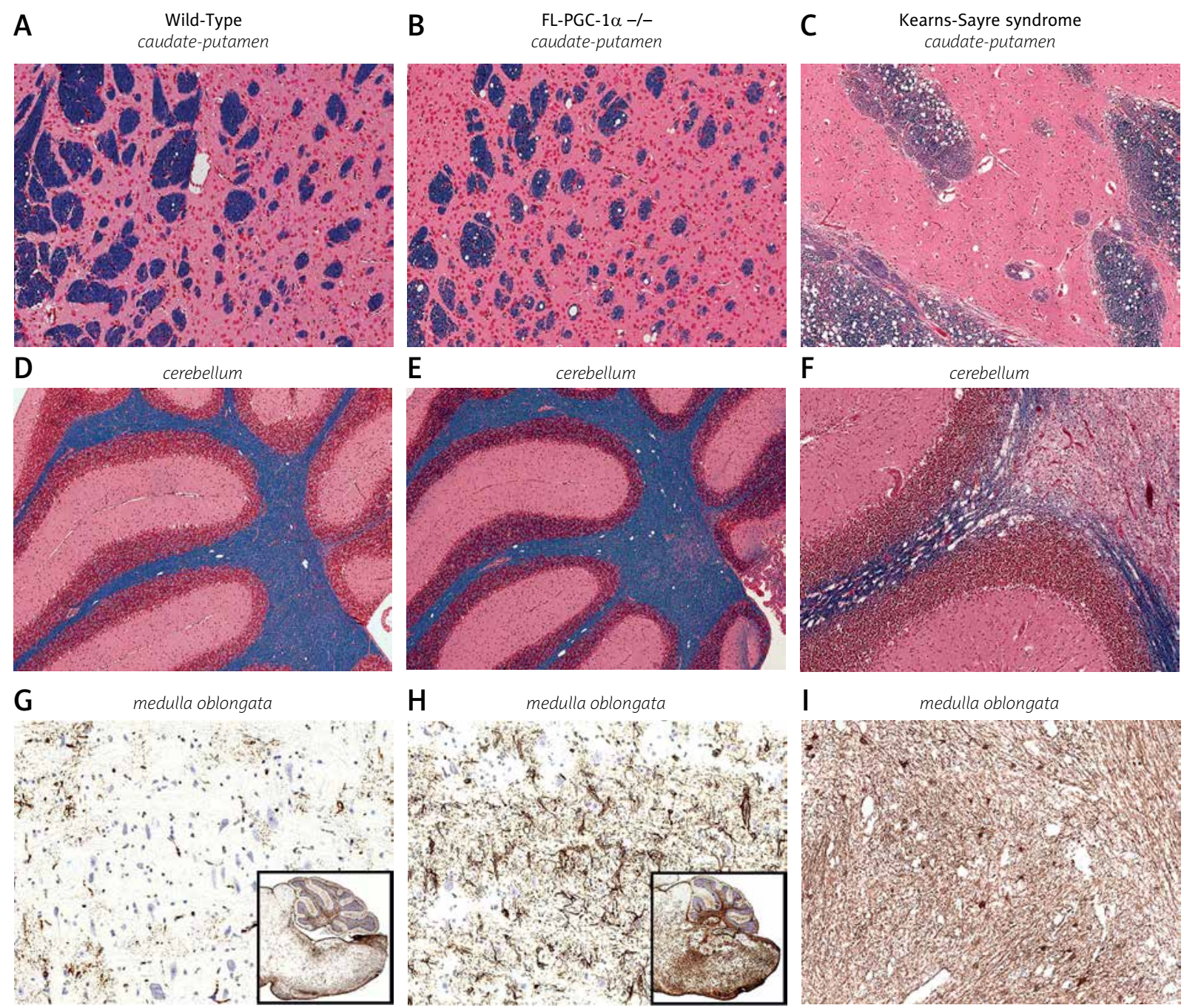

Fig. 1. Vacuolation and astroglial reaction. Aged FL-PGC-1 $\alpha$-deficient mice develop moderate to severe vacuolation ( $\mathbf{B}$ and $\mathbf{E}$ ) in areas corresponding to severe to devastating vacuolation in KSS ( $\mathbf{C}$ and $\mathbf{F}$ ) and mild vacuolation in aged wild-type mice (A and D; Klüver-Barrera). Vacuoles are in association with WM structures. Note the patchy areas of myelin pallor in the pencil fibres of the caudate-putamen (C) and the demyelinated cystic-necrotic lesion in the cerebellar WM in KSS (F). Reactive astrogliosis indicative of neuronal degeneration is present in the brainstem of FL-PGC- $1 \alpha$-deficient mice and the KSS case ( $\mathbf{H}$ and I; GFAP).

\section{Axonal pathology}

The intensity of axonal destruction in the KSS brain was variable and generally followed that of the vacuolar change. Only the severely affected cystic-necrotic lesions showed axonal loss or swelling, whereas most of the moderately vacuolated areas were devoid of axonal pathology (Fig. 3C), except for scattered swellings and APP-positive spheroids indicating acute-subacute impairment of axonal transport (Fig. 3F). Regions with severe axonal involvement were accompanied by reactive microgliosis
(Fig. 3I). The axons in the FL-PGC-1 $\alpha$-deficient mice were generally well preserved, and the patterns of $A P P$, neurofilament and microglia stainings were similar to that observed in wild-type (Fig. 3A-B, D-E and $\mathrm{G}-\mathrm{H})$.

\section{Characterization of vacuoles}

\section{White matter vacuoles}

Vacuoles within the WM were surrounded by rings of MBP-positive myelin in both the KSS and FL-PGC-1 $\alpha$-deficient brains (Fig. 4A). The vacuoles 

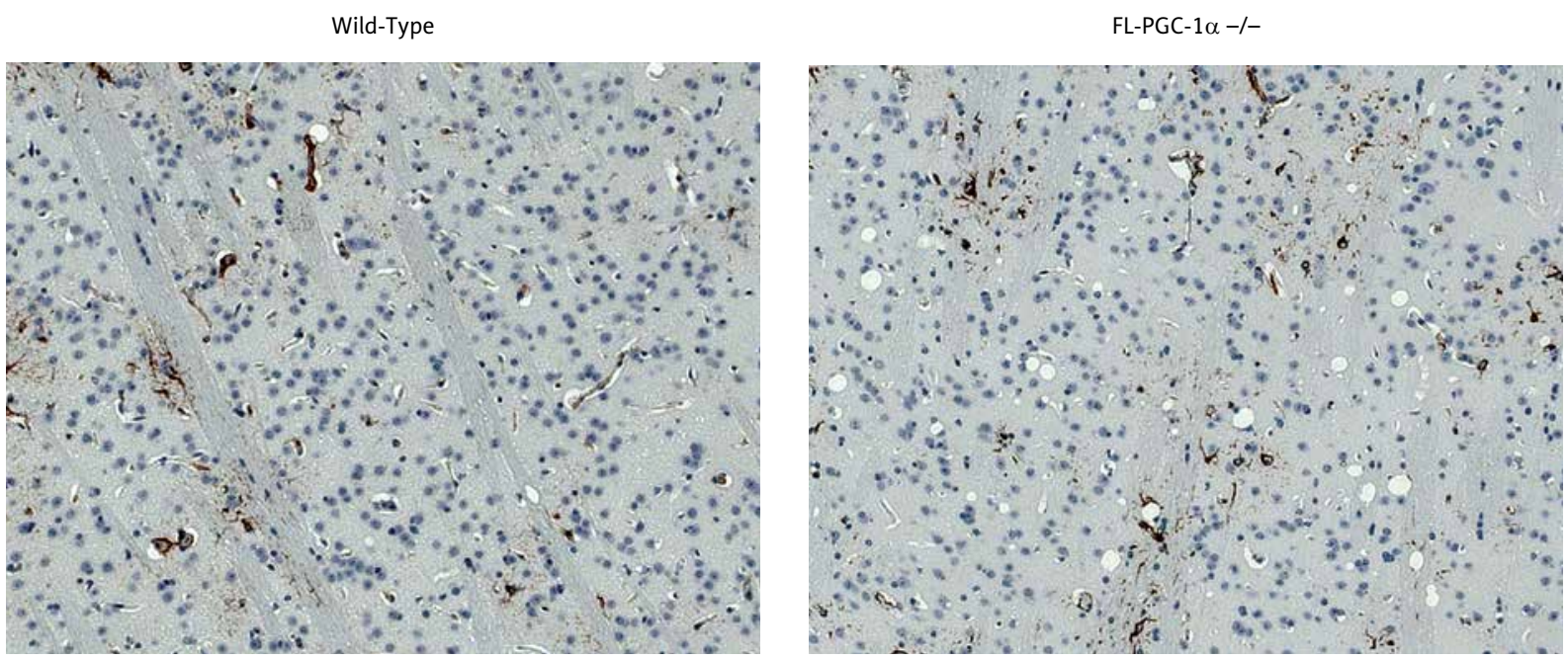

Fig. 2. The caudate-putamen of FL-PGC-1 $\alpha$-deficient mice is free of reactive astrogliosis (GFAP) - an indicator of chronic neuronal degeneration - even in this aged population, suggesting that this murine strain is not a model for Huntington's disease, as it was previously suggested.

were usually ovoid with their longitudinal axis paralleling the direction of axons. They often formed chain-like structures in longitudinal or sieve-like lesions in transverse sections. No apparent macrophage activity was present even in the areas of severe vacuolation, and no signs of active myelin degradation could be detected by Oil Red $O$ (not shown). Electron microscopy revealed that myelin 'bubbles' in the WM were formed by splitting at the intraperiod lines, and occasionally between the axons and the innermost myelin lamellae (adaxonal vacuoles); the vacuoles were 'empty' or contained various amount of debris with myelin-like figures (Fig. 5A-B, Fig. 6).

\section{Neuropil vacuoles}

Staining for neuronal (MAP-2 and SMI-32) or astrocytic (GFAP) antigens sparsely revealed associations of neuropil vacuoles with these cell-types in either the KSS or the FL-PGC-1 $\alpha$-deficient brains (Fig. 7). Staining for MBP, however, unveiled that the vast majority of neuropil vacuoles were clearly encompassed by a myelin-positive rim, suggesting the same intramyelinic localization as for vacuoles within the WM (Fig. 4B). Neuropil vacuoles were also frequently associated with oligodendrocytes in sections immunostained for TPPP/p25 (Fig. 4C). Such close contacts of oligodendrocytes and vacuoles were also observed by electron microscopy. Furthermore, vacuoles (sometimes multiloculated) could frequently be identified within the cytoplasm of glial cells, which, due to the lack of glial fibres observed in the cytoplasm, also appeared to be oligodendrocytic (Fig. 5C). Likewise myelin 'bubbles', these membrane-bound vacuoles often contained myelin-like figures, and they occasionally coalesced occupying most of the oligodendroglial cytoplasm and lead to the swelling of the cells (Fig. 5C). These observations were seen in both the KSS and the FL-PGC-1 $\alpha$-deficient brains.

\section{Discussion}

In addition to its central role in mitochondrial encephalopathies, mitochondrial dysfunction has been associated with various neurodegenerative CNS disorders [17], and is supposed to play important roles in the pathogenesis of WM lesioning in multiple sclerosis (MS) [7] as well in the aging brain [38]. Though the exact mechanism of vacuole formation in mitochondrial encephalopathies is not yet revealed, intramyelin WM vacuoles are presumed to develop due to intramyelin oedema secondary to mitochondrial dysfunction of oligodendrocytes, manifesting in splitting of the myelin sheath at the intraperiod line $[32,40,46]$. On the other hand, GM neuropil vacuoles are presumed to develop due to ion transport disorder of astrocytic membranes $[32,40,46]$.

Revisiting the above concepts of vacuole formation in conditions associated with mitochondrial 

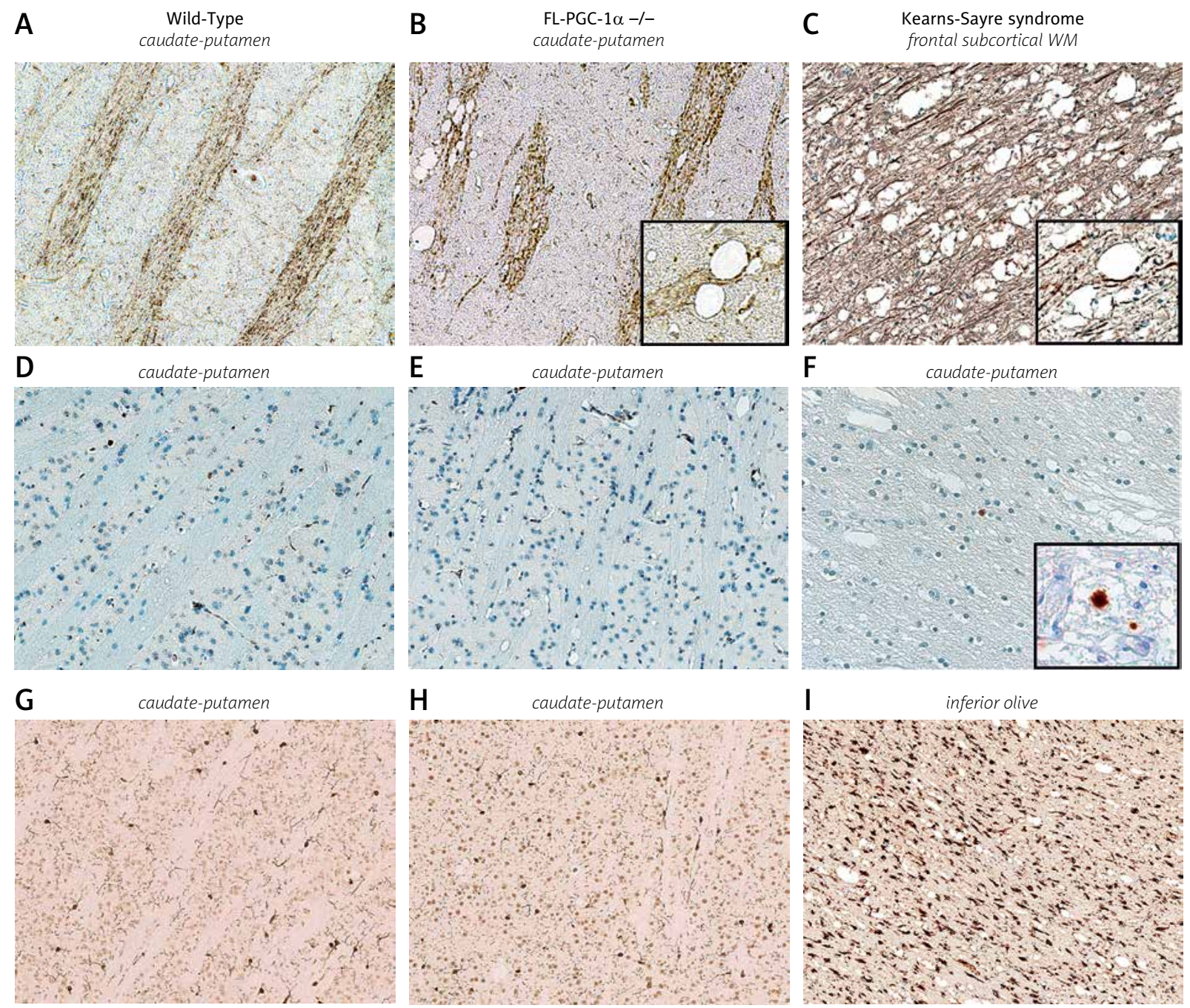

Fig. 3. Axonal pathology. Axons in areas with moderate vacuolation in KSS were relatively intact with sparse presence of axonal swellings (C; SMI-31). Such areas also showed scattered appearence of APP+ axonal spheroids indicative of subacute axonal transport impairment in KSS (F; APP). Regions with cystic-necrotic lesions showed robust microglia accumulation in KSS (I; Iba). These pathologies were virtually absent in the FL-PGC-1 $\alpha$-deficient mice and the immunohistochemical patterns were rather similar to that in aged wildtypes (A-B, D-E and G-H). Note the well-preserved axons dodging between multiple vacuoles (B), similarly to that seen in KSS $(\mathbf{C})$.

dysfunction, our study provided two main novelties. A major finding of our study is that it demonstrates commonalities of vacuolation in the WM and GM, placing oligodendrocytes in the centre of disease pathogenesis. Indeed, our observation that the vast majority of GM neuropil vacuoles are clearly myelinbound in both the KSS and FL-PGC-1 $\alpha$-deficient brains indicates that the cellular localization and thus the mechanism of vacuole-formation is most likely similar, irrespective of whether they are in WM or GM. This observation can explain the phenomenon widely observed in mitochondrial encephalopathies - including PGC-1 $\alpha$-deficient mice - that cortical vacuoles show a predilection toward the deeper layers of the neocortex, as the density of myelinated fibres apparently gradually decreases by approaching the superficial layers. Importantly, this could also explain the predilection of GM neuropil vacuoles toward the reticular area of the brainstem, thalamus, deep cerebellar nuclei, and basal ganglia adjacent to the internal capsule, as these GM regions are intermingled with the WM. Interestingly, these regions are 
FL-PGC-1 $\alpha-/-$

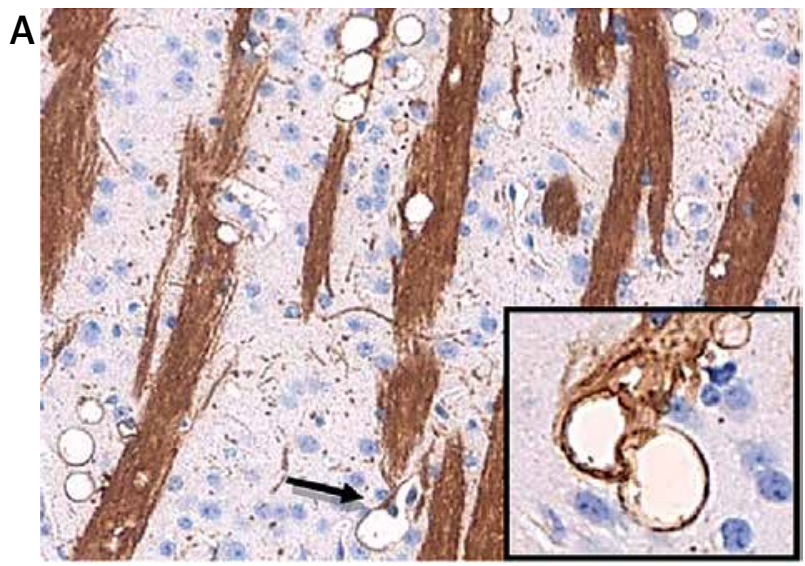

\section{B}
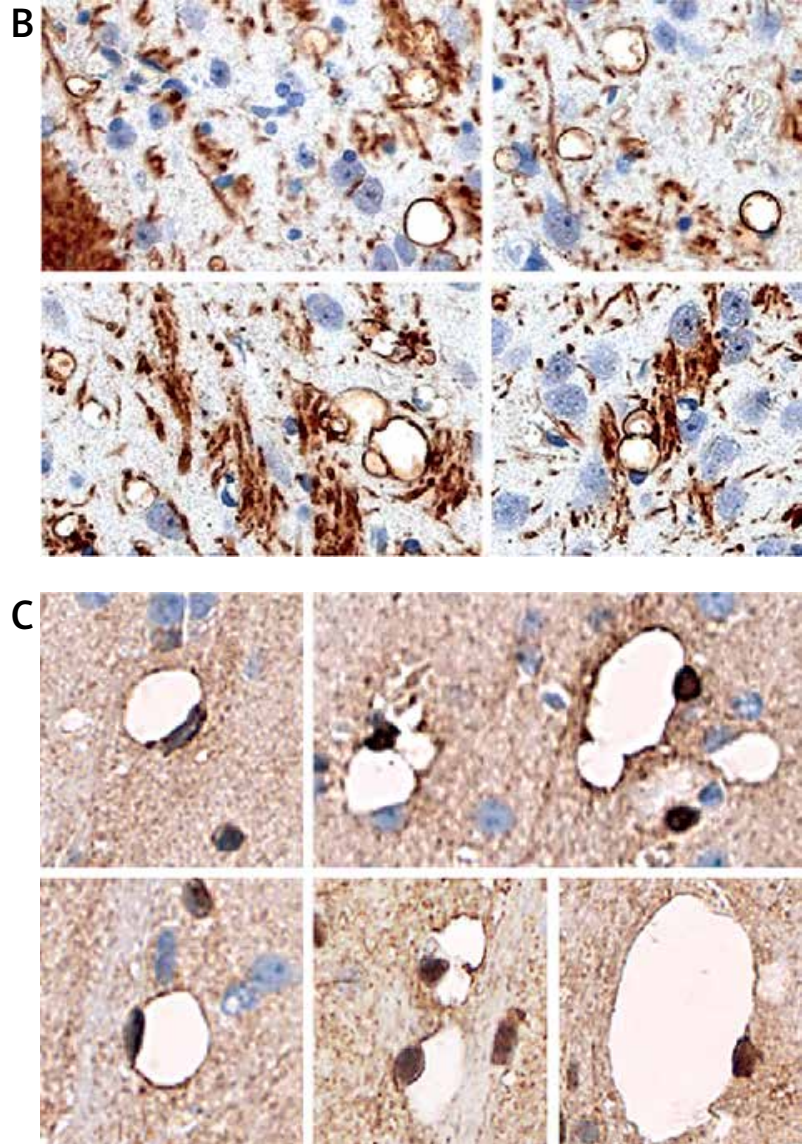

Kearns-Sayre syndrome
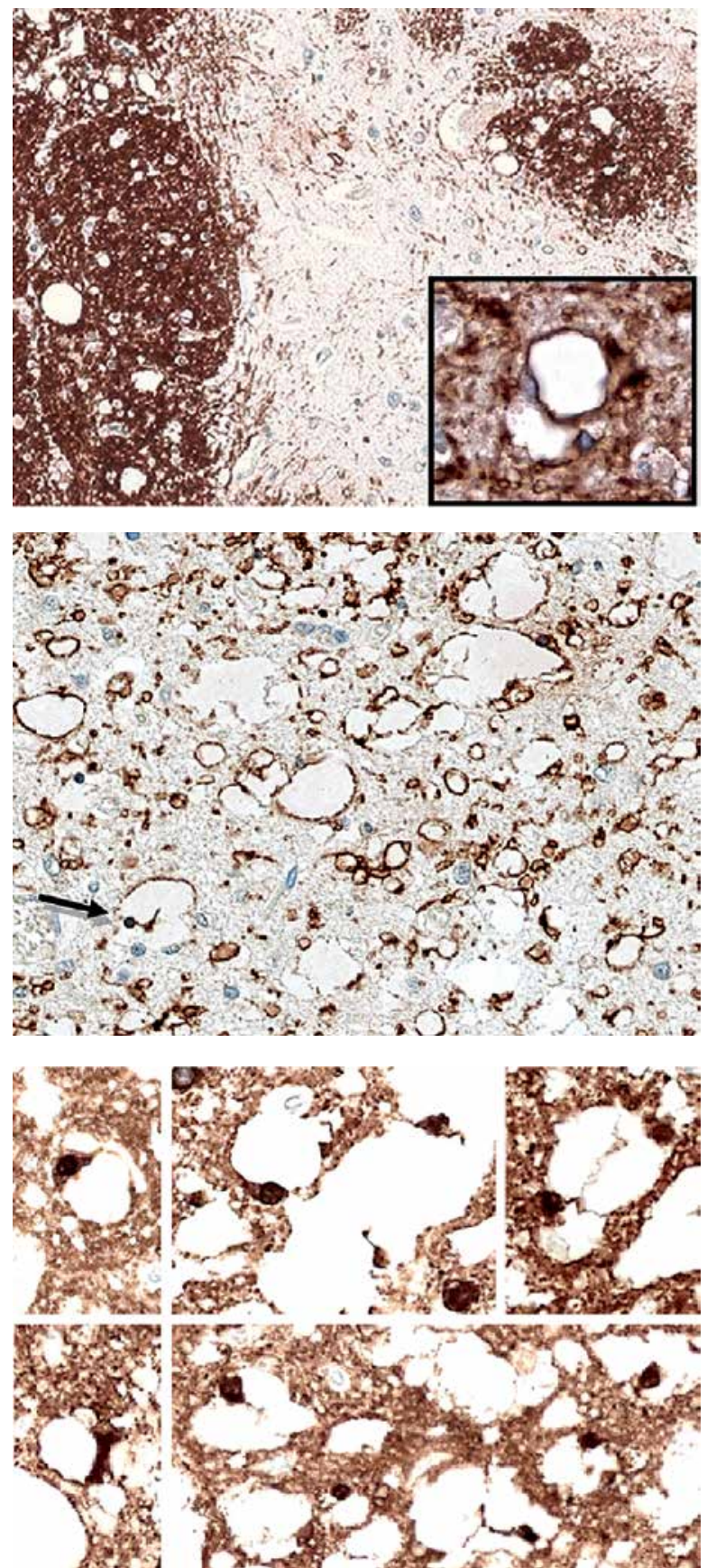

Fig. 4. Immunohistochemical characterization of vacuoles. Vacuoles within the WM are surrounded by rings of myelin (A; MBP). Likewise WM myelin 'bubbles', vacuoles within the GM neuropil are also encompassed by a myelin-positive rim, suggesting an identical origin (B; MBP). Oligodendroglial cells in close contact with and/or localized within the inner edge of either single or multiloculated vacuoles can frequently be detected by immunohistochemistry (C; TPPP/p25), suggesting an important role of oligodendrocytes in vacuole formation. Note some of the larger vacuoles being separated to multiple chambers by oligodendroglial processes $(\mathbf{C})$. Note also the glial cell encompassing a vacuole with its $\mathrm{MBP}+$ process (indicated by arrows, A - left, B - right). 
FL-PGC-1 $\alpha-/-$

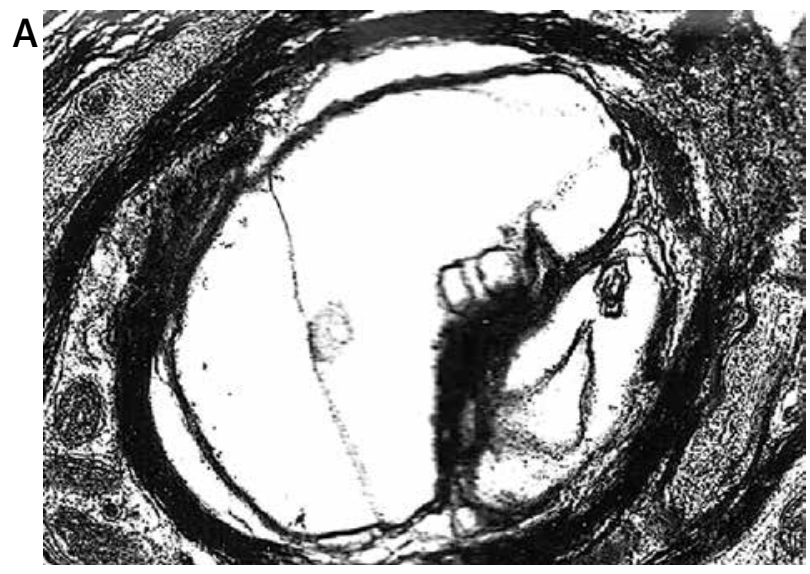

B

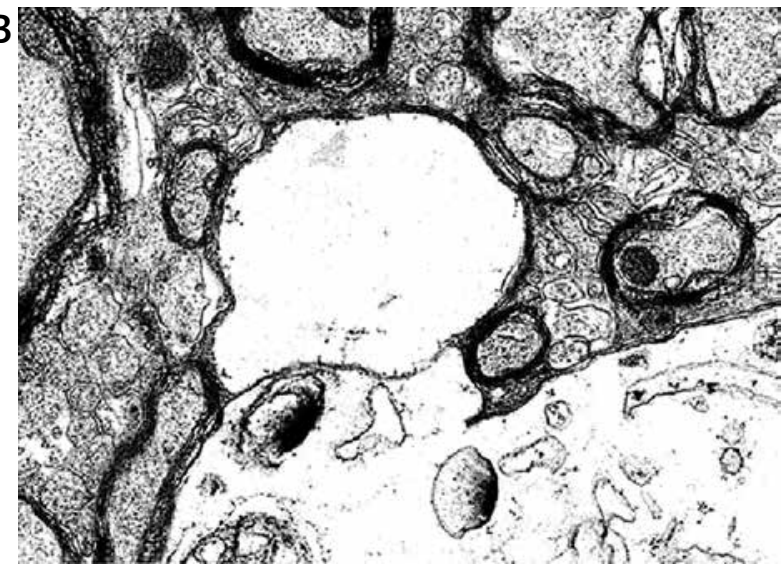

C

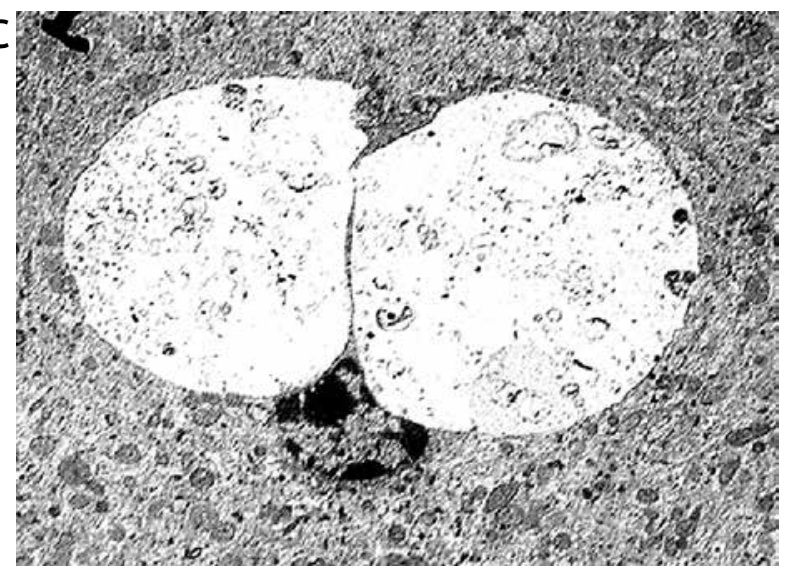

Kearns-Sayre syndrome
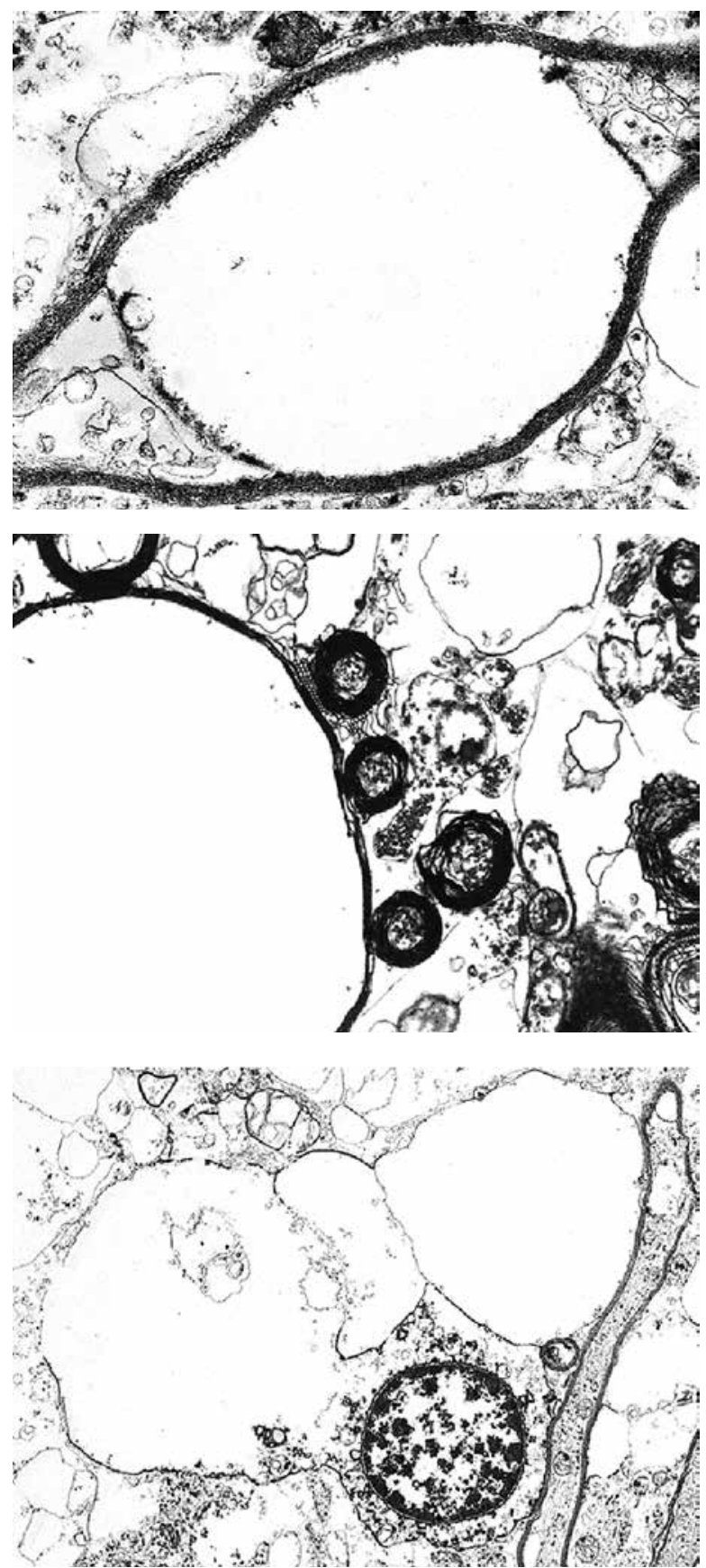

Fig. 5. Ultrastructural characterization of vacuoles. Intramyelin vacuolation can be identified also by electron microscopy (longitudinal section, A; transverse section, B). In accordance with immunohistochemical findings, vacuoles, often multiloculated, can frequently be identified within the cytoplasm of oligodendroglial cells by electron microscopy (C).

the predilection areas for the development of GM vacuolation in hepatic encephalopathy as well [52]. Considering this overlapping predilection of spongy change between hepatic and mitochondrial enceph- alopathies, and that mitochondrial disorders, including PGC-1 $\alpha$ deficiency [23], also present with hepatic involvement, the contribution of liver insufficiency to the development of mitochondrial status spongio- 
sus cannot be excluded either. Notably, intramyelin vacuolation has been frequently described in other experimental, veterinary and human metabolic conditions (Table I), suggesting that this change might be a general response to various insults that compromise the metabolism of the myelin sheath and/ or oligodendrocytes.

As a second novelty, the ultrastructural analysis revealed the common appearance of intracellular, often multiloculated formation of vacuoles within oligodendroglial cells both in the KSS case and the FL-PGC-1 $\alpha$-deficient mice. This finding was supported by the immunohistochemical observation of TPPP/p25-positive oligodendrocytes directly attaching to and sometimes bulging into vacuoles within the neuropil. The finding, however, that some vacuoles were observed closely attached to and partly encompassed by neuronal structures in MAP-2 and SMI-32 stainings suggests that at least a small proportion of vacuoles within the neuropil can be of neuronal origin, and that the mechanisms which lead to excessive intracellular membrane-bound fluid accumulation may affect different cell-types within the CNS, though to different extents. This hypothesis is supported by the report on CNS vacuoles observed also in neuron-specific PGC-1 $\alpha$ knockout mice [25].

Interestingly, though intracellular oligodendroglial vacuoles have not been previously described in mitochondrial diseases, their presence is not unprecedented in pathologies associated with mitochondrial dysfunction and/or severe cellular stress. Indeed, chronic feeding of mice with cuprizone, a copperchelating mitochondrial toxin associated with megamitochondria [41] and alterations in complex IV and superoxide dismutase (SOD) activity [1], evokes a CNS pathology comprising vacuole formation in the pons, midbrain, thalamus, cerebral and cerebellar WM, as well as in deep cortical layers [42]. It was described that cuprizone-induced intramyelin vacuolation was due to splitting at the intraperiod line and were not stained by Sudan IV (equivalent with Oil Red $O$ in our study) [42]. Additionally, the authors reported the presence of oligodendrocytes with enlarged cytoplasm containing multiloculated vesicles, and that some of these cells were juxtaposed to myelin sheaths, where a thin myelin layer appeared to form the glial membrane [42]. These findings are strikingly similar to those observed in our study. A further similarity between cuprizone-induced and mitochondrial status spongiosus is that cuprizone

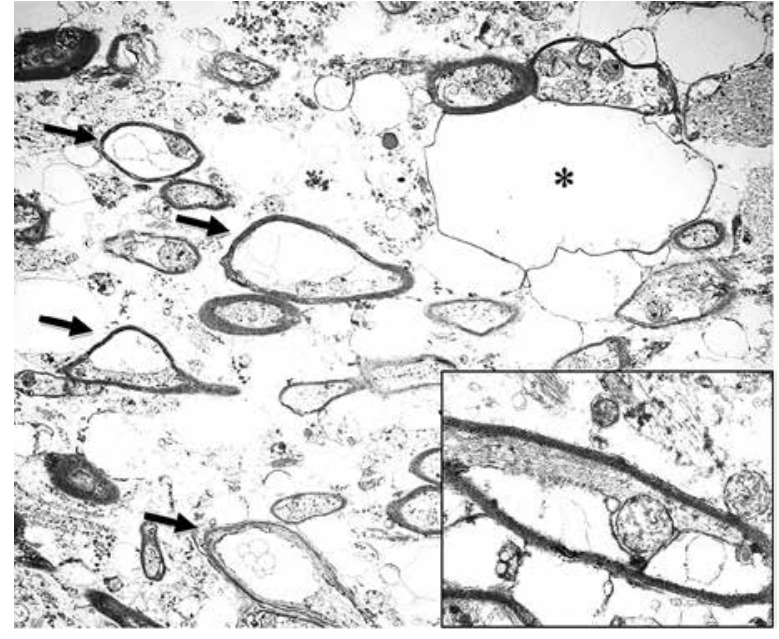

Fig. 6. Distinct types of intramyelin vacuoles in Kearns-Sayre syndrome (KSS). Asterisk denotes a huge 'classical' intramyelin vacuole originating from splitting at the intraperiod line. Arrows indicate the multiple presence of adaxonal vacuoles, some of which appear to originate from splitting between the two innermost myelin lamellae at higher power. The frequent appearance of adaxonal vacuoles may underpin the role of intra-oligodendroglial vacuolar change in the development of intramyelin vacuoles in mitochondrial encephalopathies such as KSS.

toxicity in doses evoking demyelination associates with oligodendroglial apoptosis [1], a phenomenon recently described in KSS [22]. Our observation of intra-oligodendroglial vacuolation in mitochondrial encephalopathy expands the spectrum of disorders where this has been described (Table II).

Though the neuropathological alterations in MS are results of a complex aetiology including autoimmunity, demyelination, neuronal and axonal degeneration, the central role of oligodendrocytes has also been suggested [15], which gives our observations a wider implication. The interrelation between mitochondrial leukoencephalopathies and MS is well exemplified by the fact that the above mentioned cuprizone intoxication, presenting with a highly similar neuropathology to that observed in KSS and FL-PGC-1 $\alpha$-deficient mice in our study, is in fact a widely applied toxin model of MS $[1,27]$. It has also been suggested that though a moderate mitochondrial dysfunction alone may not cause selective demyelination directly, its effect to evoke a disintegrated myelin structure may expose the sheath to 
FL-PGC-1 $\alpha-/-$
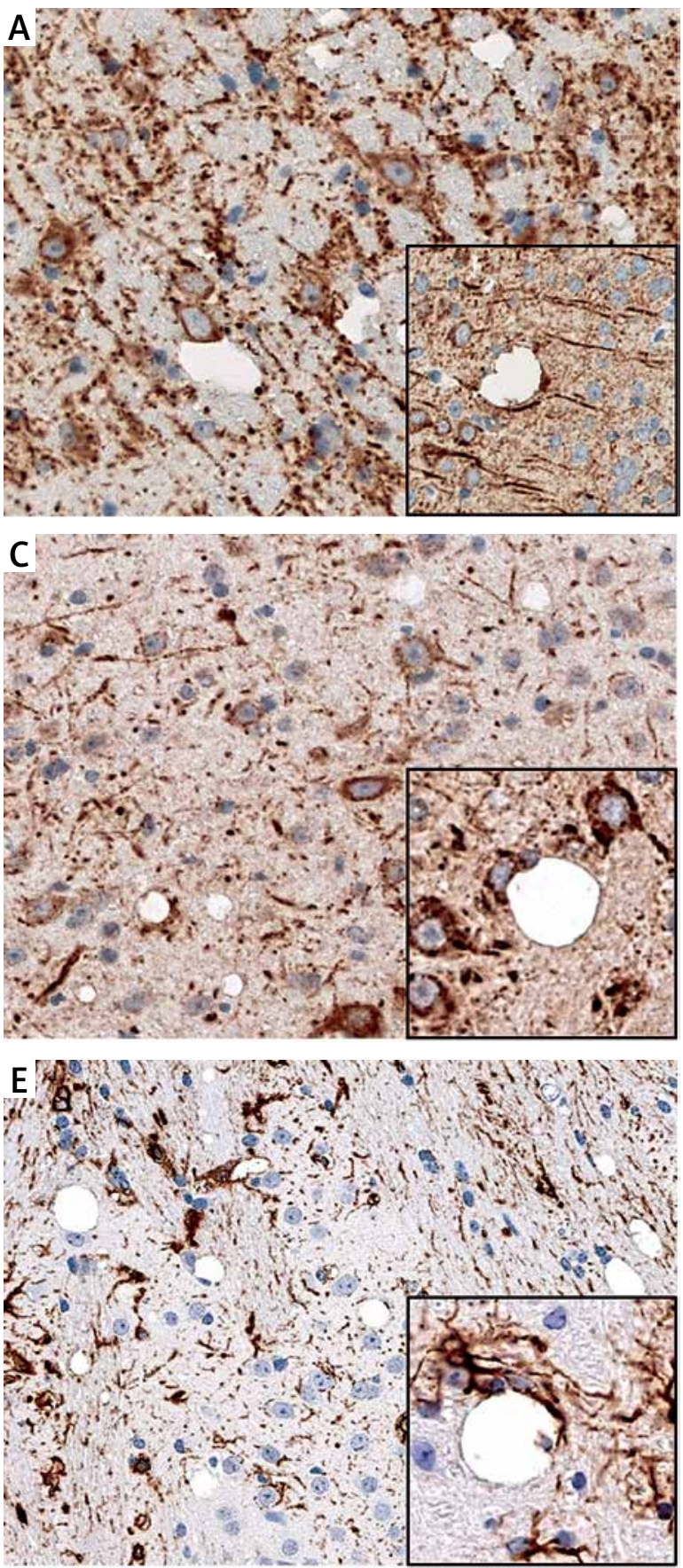

Kearns-Sayre syndrome
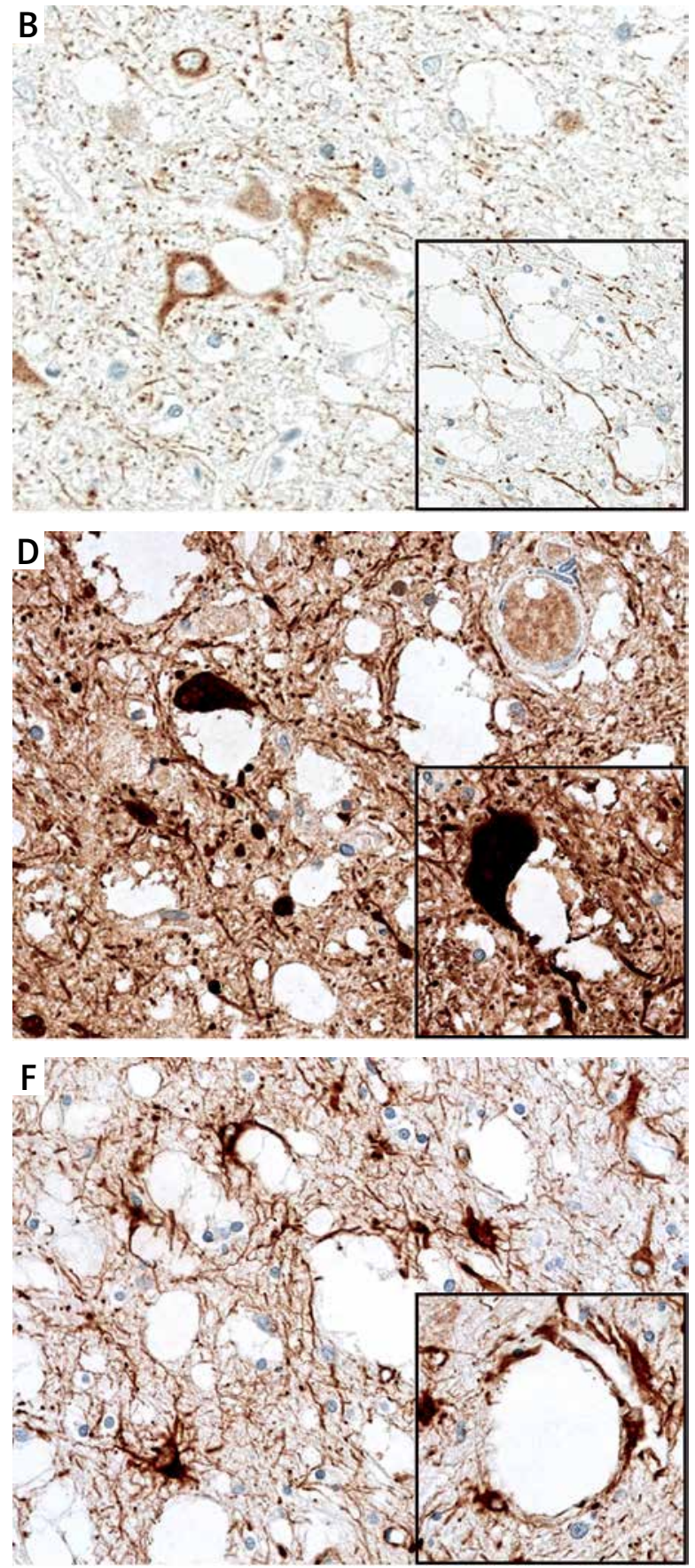

Fig. 7. Rare findings of vacuoles being in close contact with structures positive for staining against neuronal (A-B, MAP-2; C-D, SMI-32) or astroglial (E-F, GFAP) antigens. Notably, the vast majority of GM neuropil vacuoles have no immunohistochemically detectable associations with such structures, which are often merely pushed by vacuoles of most probably distinct origin.

further damage, e.g. by complex immune-mediated mechanisms [20]. Indeed, mtDNA mutations have been proposed to affect the CNS on a common metabolic basis, which may occasionally aggravate or initiate autoimmune pathology leading to MS-like lesions [20].

Besides demyelinating WM disease, our study also has implications for understanding WM lesions 
Table I. Animal and human pathologies with intramyelin vacuolation

\begin{tabular}{|c|c|}
\hline Experimental pathologies & Reference \\
\hline \multicolumn{2}{|l|}{ Intoxication by } \\
\hline actinomycin D & {$[36]$} \\
\hline copper & {$[16]$} \\
\hline cuprizone & [33], [42] \\
\hline ethidium bromide & {$[50]$} \\
\hline hexachlorophene & {$[18]$} \\
\hline isonicotinic acid hydrazide & {$[21]$} \\
\hline lysolecithin & [5] \\
\hline triethyltin & [2] \\
\hline \multicolumn{2}{|l|}{ Genetic models of } \\
\hline mitochondrial dysfunction & reviewed in [43] \\
\hline altered proteolipid expression & [3] \\
\hline Models of scrapie & {$[24],[33]$} \\
\hline \multicolumn{2}{|c|}{ Veterinary pathologies of various aetiology } \\
\hline $\begin{array}{l}\text { Helichrysum poisoning in sheep and } \\
\text { a goat }\end{array}$ & [49] \\
\hline Tetrapterys poisoning in sheep & {$[35]$} \\
\hline Maple syrup urine disease in calves & {$[31]$} \\
\hline $\begin{array}{l}\text { Hereditary spongy degeneration } \\
\text { of dogs }\end{array}$ & [29], [53] \\
\hline $\begin{array}{l}\text { Hereditary spongy degeneration } \\
\text { of silver foxes }\end{array}$ & [13] \\
\hline \multicolumn{2}{|l|}{ Human pathologies } \\
\hline Aging & reviewed in [34] \\
\hline $\begin{array}{l}\text { Aspartoacylase deficiency } \\
\text { (Canavan's disease) }\end{array}$ & reviewed in [12] \\
\hline Hepatic encephalopathy & reviewed in [12] \\
\hline $\begin{array}{l}\text { Heroin-induced spongiform } \\
\text { leukoencephalopathy }\end{array}$ & {$[51]$} \\
\hline Maple syrup urine disease & reviewed in [12] \\
\hline Mitochondrial disorders & reviewed in [12] \\
\hline Urea cycle disorders & reviewed in [12] \\
\hline
\end{tabular}

in the aging brain, a frequent pathology which includes the formation of intramyelin 'balloons' due to intraperiod line splitting [34], similar to that seen in mitochondrial disorders. Our observation that aged wild-type mice also develop vacuoles to an apparently slighter extent but at the same predilection areas as their FL-PGC- $1 \alpha$-deficient counterparts recapitulates previous observations [10]. Based on these, we propose that vacuole formation in mitochondrial encephalopathies and their representative animal models (e.g. PGC-1 $\alpha$-deficient mice) might
Table II. Experimental and veterinary pathologies with oligodendroglial vacuolation

\begin{tabular}{|lc|}
\hline Experimental pathologies & Reference \\
\hline Cuprizone intoxication & {$[42]$} \\
\hline lonizing radiation & {$[26]$} \\
\hline Methionine sulfoximine intoxication & {$[14]$} \\
\hline Triethyltin intoxication in quaking mice & {$[28]$} \\
\hline Twitcher mouse & {$[45]$} \\
\hline Zitter rat & {$[19]$} \\
\hline Veterinary pathologies & \\
\hline Hereditary ataxia of the rabbit & {$[30]$} \\
\hline $\begin{array}{l}\text { Hereditary spongy degeneration of silver } \\
\text { foxes }\end{array}$ & {$[13]$} \\
\hline
\end{tabular}

also be regarded as an accelerated form of 'normal' WM degeneration, which underpins the role of (oligodendroglial) mitochondrial dysfunction in aging.

Although the potential role of oligodendrocytes in WM vacuolation in mitochondrial encephalopathies has already been suggested, the fundamental concepts included (1) a disrupted ion-homeostasis of the sheath, (2) a dysfunction of the blood-brain barrier, in both cases with consequent development of 'intramyelin oedema' $[32,40,46]$. These hypotheses, however, do not explain why vacuoles develop focally and how multiple vacuoles can be found within the same internode, instead of a complete splitting and diffuse loosening of the sheath between all lamellae. We propose that chronic mitochondrial dysfunction in a yet unknown pathway leads to the formation of multiple intra-oligodendroglial fluid-filled vacuoles. Increased intracellular content might provoke splitting between the intracellular surfaces of the myelin sheath (major dense line). Due to their firm connections at the macromolecular level, this would cause tears and focal myelin disruptions, allowing the vacuolar content to access into the virtual space between the loosely attached extracellular surfaces (intraperiod lines). Consequently, this could evoke the formation of focal splits and eventually myelin bubbles. Accordingly, disruption of the lateral loops would result in intraperiod line splitting at the corresponding levels, whereas leakage from the inner tongue would cause adaxonal swelling between the axolemma and the innermost myelin lamellae or between the two innermost layers. Supporting 
this theoretical consideration, such distinct types of intramyelin vacuoles have indeed been described in experimental status spongiosus [36,50], and could also be observed in our study (Fig. 6).

\section{Conclusions}

In addition to a detailed comparative neuropathological characterization of a recently proposed murine model of mitochondrial encephalopathy and human KSS, this study first provides morphological evidence for the identical intramyelinic nature of WM and GM vacuolation as well as for the presence of intra-oligodendroglial vacuoles in mitochondrial disease, which may have a pathogenetic role in the development of intramyelin vacuoles, providing a possible source of focal myelin splitting. Our observations place oligodendrocytes in the centre of the pathogenesis of CNS lesioning in association with chronic mitochondrial dysfunction both in WM and $G M$, which is in line with the recognition that oligodendrocytes, contrasting astrocytes [11], are most sensitive to mitochondrial stress, exceeding the vulnerability of neurons [8]. This may serve as rationale for cytoprotective targeting of the oligodendrocytes in mitochondrial encephalopathies as well as in other disorders with vacuole formation and myelin degeneration.

\section{Acknowledgements}

The study was supported by the Hungarian Brain Research Program - Grant No. KTIA_13_NAP-A-II/18., the European Union and the State of Hungary, cofinanced by the European Social Fund in the framework of TÁMOP 4.2.4. A/2-11-1-2012-0001 'National ExcellenceProgram',TÁMOP-4.2.2/B-10/1-2010-0012, and TÁMOP-4.2.2.A-11/1/KONV-2012-0052. P.P.L. is supported by OEAD and Healthy Ageing Research Centre project (REGPOT-2012-2013-1, 7FP). P.P.L. and G.G.K. are supported by ÖAD Austria-Poland (PL 04/2014). We are grateful to Agnes Herczegfalvi, Albert C. Ludolph and Patrick Weydt for their valuable contribution.

\section{Disclosure}

Authors report no conflict of interest.

\section{References}

1. Acs P, Selak MA, Komoly S, Kalman B. Distribution of oligodendrocyte loss and mitochondrial toxicity in the cuprizone-in- duced experimental demyelination model. J Neuroimmunol 2013; 262: 128-131.

2. Aleu FP, Katzman R, Terry RD. Fine structure and electrolyte analyses of cerebral edema induced by alkyl tin intoxication. J Neuropathol Exp Neurol 1963; 22: 403-413.

3. Anderson TJ, Schneider A, Barrie JA, Klugmann M, McCulloch MC, Kirkham D, Kyriakides E, Nave KA, Griffiths IR. Late-onset neurodegeneration in mice with increased dosage of the proteolipid protein gene. J Comp Neurol 1998; 394: 506-519.

4. Betts J, Lightowlers RN, Turnbull DM. Neuropathological aspects of mitochondrial DNA disease. Neurochem Res 2004; 29: 505-511.

5. Blakemore WF. Observations on remyelination in the rabbit spinal cord following demyelination induced by lysolecithin. Neuropathol Appl Neurobiol 1978; 4: 47-59.

6. Brown GK, Squier MV. Neuropathology and pathogenesis of mitochondrial diseases. J Inherit Metab Dis 1996; 19: 553-572.

7. Campbell GR, Worrall JT, Mahad DJ. The central role of mitochondria in axonal degeneration in multiple sclerosis. Mult Scler 2014; 20: 1806-1813.

8. Dewar D, Underhill SM, Goldberg MP. Oligodendrocytes and ischemic brain injury. J Cereb Blood Flow Metab 2003; 23: 263-274.

9. Filosto M, Tomelleri G, Tonin P, Scarpelli M, Vattemi G, Rizzuto N, Padovani A, Simonati A. Neuropathology of mitochondrial diseases. Biosci Rep 2007; 27: 23-30.

10. Fraser $H$. Neuropathology of scrapie: the precision of the lesions and their significance. In: Prusiner SB, Hadlow WJ (eds.). Slow Transmittible Diseases of the Nervous System. Academic Press, New York 1979; pp. 397-406.

11. Goldberg MP, Choi DW. Combined oxygen and glucose deprivation in cortical cell culture: calcium-dependent and calciumindependent mechanisms of neuronal injury. J Neurosci 1993; 13: 3510-3524.

12. Greenfield JG, Love S, Louis DN, Ellison D. Greenfield's neuropathology. $8^{\text {th }}$ ed. Hodder Arnold, London 2008.

13. Hagen G, Blakemore WF, Bjerkås I. Ultrastructural findings in spongy degeneration of white matter in silver foxes (Vulpes vulpes). A naturally occurring demyelinating disease with oligodendrocyte vacuolation. Acta Neuropathol 1990; 80: 590-596.

14. Harris B. Cortical alterations due to methionine sulfoximine. Ultrastructure during seizure activity. Arch Neurol 1964; 11: 388-407.

15. Höftberger R, Fink S, Aboul-Enein F, Botond G, Olah J, Berki T, Ovadi J, Lassmann H, Budka H, Kovacs GG. Tubulin polymerization promoting protein (TPPP/p25) as a marker for oligodendroglial changes in multiple sclerosis. Glia 2010; 58: 1847-1857.

16. Howell JM, Blakemore WF, Gopinath C, Hall GA, Parker JH. Chronic copper poisoning and changes in the central nervous system of sheep. Acta Neuropathol 1974; 29: 9-24.

17. Johri A, Beal MF. Mitochondrial dysfunction in neurodegenerative diseases. J Pharmacol Exp Ther 2012; 342: 619-630.

18. Kanno T, Sasaki S, Yamada N, Kawasako K, Tsuchitani M. Hexachlorophene and cuprizone induce the spongy change of the developing rat brain by different mechanisms: the role of 2', 3'-cyclic 
nucleotide 3'-phosphodiesterase (CNPase). J Vet Med Sci 2012; 74 837-843.

19. Kondo A, Sendoh S, Miyata K, Takamatsu J. Spongy degeneration in the zitter rat: ultrastructural and immunohistochemical studies. J Neurocytol 1995; 24: 533-544.

20. Kovács GG, Höftberger R, Majtényi K, Horváth R, Barsi P, Komoly S, Lassmann H, Budka H, Jakab G. Neuropathology of white matter disease in Leber's hereditary optic neuropathy. Brain 2005; 128: 35-41.

21. Lampert PW, Schochet SS. Electron microscopic observations on experimental spongy degeneration of the cerebellar white matter. J Neuropathol Exp Neurol 1968; 27: 210-220.

22. Lax NZ, Campbell GR, Reeve AK, Ohno N, Zambonin J, Blakely EL, Taylor RW, Bonilla E, Tanji K, DiMauro S, Jaros E, Lassmann H, Turnbull DM, Mahad DJ. Loss of myelin-associated glycoprotein in kearns-sayre syndrome. Arch Neurol 2012; 69: 490-499.

23. Leone TC, Lehman JJ, Finck BN, Schaeffer PJ, Wende AR, Boudina S, Courtois M, Wozniak DF, Sambandam N, Bernal-Mizrachi C, Chen Z, Holloszy JO, Medeiros DM, Schmidt RE, Saffitz JE, Abel ED, Semenkovich CF, Kelly DP. PGC-1alpha deficiency causes multi-system energy metabolic derangements: muscle dysfunction, abnormal weight control and hepatic steatosis. PLoS Biol 2005; 3: e101.

24. Liberski PP, Yanagihara R, Wells GA, Gibbs CJ Jr, Gajdusek DC. Ultrastructural pathology of axons and myelin in experimental scrapie in hamsters and bovine spongiform encephalopathy in cattle and a comparison with the panencephalopathic type of Creutzfeldt-Jakob disease. J Comp Pathol 1992; 106: 383-398.

25. Ma D, Li S, Lucas EK, Cowell RM, Lin JD. Neuronal inactivation of peroxisome proliferator-activated receptor gamma coactivator 1alpha (PGC-1alpha) protects mice from diet-induced obesity and leads to degenerative lesions. J Biol Chem 2010; 285: 39087-39095.

26. Maxwell DS, Kruger L. The reactive oligodendrocyte. An electron microscopic study of cerebral cortex following alpha particle irradiation. Am J Anat 1966; 118: 437-459.

27. Miron VE, Kuhlmann T, Antel JP. Cells of the oligodendroglial lineage, myelination, and remyelination. Biochim Biophys Acta 2011; 1812: 184-193.

28. Nagara H, Suzuki K, Tiffany CW, Suzuki K. Triethyl tin does not induce intramyelinic vacuoles in the cns of the quaking mouse. Brain Res 1981; 225: 413-420.

29. Neer TM, Kornegay JN. Leucoencephalomalacia and cerebral white matter vacuolar degeneration in two related Labrador retriever puppies. J Vet Intern Med 1995; 9: 100-104.

30. O'Leary JL, Harris AB, Fox RR, Smith JM, Tidwell M. Ultrastructural lesions in rabbit hereditary ataxia. Arch Neurol 1965; 13: 238-262.

31. O’Toole D, Montgomery DL, Steadman L, O'Rourke B, Russell W, Dennis J. Status spongiosus of white matter in newborn Gelbvieh-cross calves. J Vet Diagn Invest 2005; 17: 546-553.

32. Oldfors A, Fyhr IM, Holme E, Larsson NG, Tulinius M. Neuropathology in Kearns-Sayre syndrome. Acta Neuropathol 1990; 80: 541-546.

33. Pattison IH, Jebbett JN. Histopathological similarities between scrapie and cuprizone toxicity in mice. Nature 1971; 230: 115-117.

34. Peters $A$. The effects of normal aging on myelin and nerve fibers: a review. J Neurocytol 2002; 31: 581-593.
35. Riet-Correa G, Riet-Correa F, Schild AL, Barros SS, Soares MP. Abortion and neonatal mortality in sheep poisoned with Tetrapterys multiglandulosa. Vet Pathol 2009; 46: 960-965.

36. Rizzuto N, Gambetti PL. Status spongiosus of rat central nervous system induced by actinomycin D. Acta Neuropathol 1976; 36: 21-30.

37. Róna-Vörös K, Weydt P. The role of PGC-1alpha in the pathogenesis of neurodegenerative disorders. Curr Drug Targets 2010; 11: 1262-1269.

38. Santos RX, Correia SC, Zhu X, Smith MA, Moreira PI, Castellani RJ, Nunomura A, Perry G. Mitochondrial DNA oxidative damage and repair in aging and Alzheimer's disease. Antioxid Redox Signal 2013; 18: 2444-2457.

39. Scheper GC, van der Klok T, van Andel RJ, van Berkel CG, Sissler M, Smet J, Muravina TI, Serkov SV, Uziel G, Bugiani M, Schiffmann R, Krägeloh-Mann I, Smeitink JA, Florentz C, Van Coster R, Pronk JC, van der Knaap MS. Mitochondrial aspartyl-tRNA synthetase deficiency causes leukoencephalopathy with brain stem and spinal cord involvement and lactate elevation. Nat Genet 2007; 39: 534-539.

40. Sparaco M, Bonilla E, DiMauro S, Powers JM. Neuropathology of mitochondrial encephalomyopathies due to mitochondrial DNA defects. J Neuropathol Exp Neurol 1993; 52: 1-10.

41. Suzuki K. Giant hepatic mitochondria: production in mice fed with cuprizone. Science 1969; 163: 81-82.

42. Suzuki K, Kikkawa Y. Status spongiosus of CNS and hepatic changes induced by cuprizone (biscyclohexanone oxalyldihydrazone). Am J Pathol 1969; 54: 307-325.

43. Szalardy L, Zadori D, Plangar I, Vecsei L, Weydt P, Ludolph AC, Klivenyi P, Kovacs GG. Neuropathology of partial PGC-1alpha deficiency recapitulates features of mitochondrial encephalopathies but not of neurodegenerative diseases. Neurodegener Dis 2013; 12: 177-188.

44. Taft RJ, Vanderver A, Leventer RJ, Damiani SA, Simons C, Grimmond SM, Miller D, Schmidt J, Lockhart PJ, Pope K, Ru K, Crawford J, Rosser T, de Coo IF, Juneja M, Verma IC, Prabhakar P, Blaser S, Raiman J, Pouwels PJ, Bevova MR, Abbink TE, van der Knaap MS, Wolf NI. Mutations in DARS cause hypomyelination with brain stem and spinal cord involvement and leg spasticity. Am J Hum Genet 2013; 92: 774-780.

45. Takahashi H, Igisu H, Suzuki K, Suzuki K. The twitcher mouse: an ultrastructural study on the oligodendroglia. Acta Neuropathol 1983; 59: 159-166.

46. Tanji K, DiMauro S, Bonilla E. Disconnection of cerebellar Purkinje cells in Kearns-Sayre syndrome. J Neurol Sci 1999; 166: 64-70.

47. Tanji K, Kunimatsu T, Vu TH, Bonilla E. Neuropathological features of mitochondrial disorders. Semin Cell Dev Biol 2001; 12: 429-439.

48. van der Knaap MS, van der Voorn P, Barkhof F, Van Coster R, Krägeloh-Mann I, Feigenbaum A, Blaser S, Vles JS, Rieckmann P, Pouwels PJ. A new leukoencephalopathy with brainstem and spinal cord involvement and high lactate. Ann Neurol 2003; 53: 252-258.

49. van der Lugt JJ, Olivier J, Jordaan P. Status spongiosis, optic neuropathy, and retinal degeneration in Helichrysum argyro- 
sphaerum poisoning in sheep and a goat. Vet Pathol 1996; 33 : 495-502.

50. Yajima K, Suzuki K. Ultrastructural changes of oligodendroglia and myelin sheaths induced by ethidium bromide. Neuropathol Appl Neurobiol 1979; 5: 49-62.

51. Yin R, Lu C, Chen Q, Fan J, Lu J. Microvascular damage is involved in the pathogenesis of heroin induced spongiform leukoencephalopathy. Int J Med Sci 2013; 10: 299-306.

52. Zachary JF, McGavin MD. Pathologic Basis of Veterinary Dis ease. $5^{\text {th }}$ ed. Elsevier Health Sciences Mosby, St Louis 2011.

53. Zachary JF, O'Brien DP. Spongy degeneration of the central nervous system in two canine littermates. Vet Pathol 1985; 22: 561-571. 